\title{
Expression of Bone Morphogenetic Protein-6 in Dental Follicle Stem Cells and Its Effect on Osteogenic Differentiation
}

\author{
Shaomian Yao Hongzhi He Dina L. Gutierrez Maryam Rezai Rad Dawen Liu \\ Chunhong Li Michael Flanagan Gary E. Wise \\ Department of Comparative Biomedical Sciences, School of Veterinary Medicine, Louisiana State University, \\ Baton Rouge, La., USA
}

\section{Key Words}

Bone morphogenetic protein-6 $\cdot$ Dental follicle stem cells .

Differentiation · Gene expression · Osteogenesis genesis capability. Increased BMP6 expression seen in vivo in the DF may reflect the activation of DFSCs for osteogenic differentiation for bone growth during tooth eruption.

(c) 2014 S. Karger AG, Basel

\section{Introduction}

The dental follicle (DF), a loose connective tissue sac surrounding the unerupted tooth, plays a critical role in regulating tooth eruption. It has been determined that the DF produces molecules at critical times to upregulate osteoclastogenesis to promote bone resorption for the for-

\section{Abbreviations used in this paper}

BMP6 bone morphogenetic protein-6

BSP bone sialoprotein

DF dental follicle

DFC dental follicle cell

DFSC dental follicle stem cell

FBS fetal bovine serum

hrBMP6 human recombinant BMP6

OCN osteocalcin

MEM minimum essential medium

RGE relative gene expression

Runx2 runt-related transcription factor 2

siRNA short interfering RNA need to express high levels of BMP6 to maintain their osteo-

\section{KARGER}

E-Mail karger@karger.com

www.karger.com/cto
(C) 2014 S. Karger AG, Basel

$1422-6405 / 14 / 1986-0438 \$ 39.50 / 0$
Dr. Shaomian Yao

Department of Comparative Biomedical Sciences

School of Veterinary Medicine, Louisiana State University

Baton Rouge, LA 70803 (USA)

E-Mail shaomia@lsu.edu 
mation of an eruption pathway [Wise and King, 2008]. Studies suggest that tooth eruption also requires bone formation. In a surgical study, when the basal half of the DF of the dog fourth premolar was removed, no bone formation occurred in the base of the tooth crypt and the tooth did not erupt [Marks and Cahill, 1987], suggesting that the DF is essential for bone formation during tooth eruption. Studies in our lab have determined that the bone formation at the base of the tooth crypt likely serves as an eruption force to push the tooth out of its bony crypt [Wise et al., 2007, 2011]. To determine what molecules the DF uses to regulate the bone formation needed for tooth eruption, we examined the expression of bone morphogenetic proteins (BMPs) in the DF and reported the expression of BMP2, BMP3 and BMP6 [Wise and Yao, 2006; Yao et al., 2010; Wise et al., 2011]. In particular, an increase in BMP6 expression was coincident with the increase in bone formation seen in the base of the tooth crypt. BMP6 expression in the basal half of the rat DF was significantly higher than in the coronal half of the DF [Wise et al., 2011]. Furthermore, knocking down BMP6 expression by transfection of BMP6-short interfering RNA (siRNA) into the rat $\mathrm{DF}$ of the first mandibular molar resulted in reduction of bone formation at the base of the crypt of the molar and inhibited eruption of that molar [Wise et al., 2011]. This indicated that BMP6 was a key molecule to promote the osteogenesis needed for tooth eruption.

Recently, multipotent stem cells have been isolated from the DF of various species [Honda et al., 2010]. Given the fact that rat DF stem cells (DFSCs) possess osteogenic differentiation capability [Yao et al., 2008] and that the DF is located adjacent to the alveolar bone, we have proposed that DFSCs might participate in the osteogenesis for bone formation during tooth eruption. Because studies have reported the capability of BMP6 to induce osteogenic differentiation [Gruber et al., 2003; Kemmis et al., 2010], and because an increase in BMP6 expression in the DF is coincident with the bone growth at the base of the tooth crypt [Wise et al., 2011], the objectives of this study were to evaluate the BMP6 expression in DF-derived cell populations and to elucidate its role in maintaining and regulating the osteogenic capability of DFSCs. The role of DFSCs in tooth eruption is also discussed.

\section{Materials and Methods}

Culture and Differentiation of DF-Derived Cells

DFs were isolated from first mandibular molars of rat pups and digested with trypsin to obtain a cell suspension. The protocol for animal use was approved by the Institutional Animal Care and Use
Committee. For cultures of non-stem cell DF cells (DFCs), cells were incubated in minimum essential medium (MEM) supplemented with $10 \%$ newborn calf serum (Life Technologies, Grand Island, N.Y., USA) and $1 \mathrm{~mm}$ sodium pyruvate. This culture condition resulted in homogenous fibroblast-like cells all expressing vimentin [Wise et al., 1992], which is a fibroblast marker [Sappino et al., 1990]. Further studies showed that DFCs cannot be induced to differentiate into other cell types [Yao et al., 2008], indicating that the DFC population did not contain stem/stromal cells. For DFSC cultures, cells were incubated in stem cell growth medium consisting of a-MEM supplemented with $20 \%$ fetal bovine serum (FBS; Atlanta Biologicals Inc., Flowery Branch, Ga., USA). When cells reached about $90 \%$ confluence, they were detached by trypsin and passaged to fresh flasks at a 1:3 ratio until the desired passages were reached. The established cells were analyzed to determine the capability of osteogenic differentiation at passage 3. The DFSCs with strong differentiation capability and DFCs with no differentiation ability were used for subsequent experiments. For all experiments, the cells were incubated at $37^{\circ} \mathrm{C}$ in $5 \% \mathrm{CO}_{2}$ with a change of medium every 4 days.

To determine the osteogenic capability of DFSCs, DFSCs were seeded in 6-well plates at 80,000 cells/well and incubated with osteogenic induction medium consisting of Dulbecco's modified eagle medium-low glucose 10\% FBS and osteogenic induction reagents $\left(50 \mu \mathrm{g} / \mathrm{ml}\right.$ ascorbate- 2 phosphate, $10^{-5} \mathrm{mM}$ dexamethasone and $10 \mathrm{~mm} \beta$-glycerophosphate) when cells reached about $80 \%$ confluence for osteogenesis. The control DFSCs were also cultured in Dulbecco's modified eagle medium-low glucose with 10\% FBS but without addition of osteogenic induction reagents. After 2 weeks of induction, the cultures were fixed with neutral buffered formalin and stained with $1 \%$ alizarin red to detect calcium deposits. To ensure the staining was for calcium, some wells were treated with $10 \%$ EDTA prior to alizarin red staining. DFSCs were also collected for RNA isolation to determine expression of selected marker genes, bone sialoprotein (BSP) and osteocalcin (OCN), and for assessment of differentiation after induction.

\section{BMP6 Expression in DFCs and DFSCs}

DFCs and DFSCs established as described above were seeded into T-25 flasks and cultured in a-MEM supplemented with $20 \%$ FBS for 1 week. Cells were collected for RNA and protein extraction to compare the expression of BMP6 using real-time RT-PCR and Western blotting. To study the BMP6 expression during osteogenesis, DFSCs of different passages were seeded into 6-well plates and incubated in osteogenic induction medium. The cells were collected after 7 days of induction, and total RNA was extracted from the cells. Gene expression was assessed with real-time RT-PCR.

\section{Effect of BMP6 on Osteogenic Differentiation of DFSCs}

Two experiments were conducted to determine the effect of BMP6 on osteogenesis of DFSCs. For both experiments, DFSCs were seeded into 6 -well plates and grown to about $80 \%$ confluence. The first experiment was designed to determine the effect of exogenous BMP6 on osteogenesis of DFSCs. To do that, DFSCs of passages 3, 7 and 11 were induced for osteogenic differentiation with or without the presence of $80 \mathrm{ng} / \mathrm{ml}$ human recombinant BMP6 (hrBMP6) purchased from PeproTech (Rocky Hill, N.J., USA) in osteogenic induction medium. Alizarin red staining was conducted after 2-3 weeks of induction. The staining was analyzed using 
Table 1. Primers used in this study

\begin{tabular}{|c|c|c|}
\hline Gene symbol & Gene bank accession No. & Sequence \\
\hline BMP6 & NM_013107 & $\begin{array}{l}\text { F: 5'-CTTACA GGAGCATCAGCACAGA-3' } \\
\text { R: 5'-GTCACCACCCA CAGATTGCTA-3' }\end{array}$ \\
\hline BSP & AB001383 & $\begin{array}{l}\text { F: } 5^{\prime} \text {-ACGCTGGAAAGTTGGAGTTAGCTG-3' } \\
\text { R: 5'-TTCCTCTTCCTCGTCGCTTTCCTT-3' }\end{array}$ \\
\hline OCN & M23637 & $\begin{array}{l}\text { F: 5'-ACTGCATTCTGCCTCTCTGACCT-3' } \\
\text { R: } 5^{\prime} \text {-TATTCACCACCTTACTGCCCTCCT-3' }\end{array}$ \\
\hline F-Spondin & M88469 & $\begin{array}{l}\text { F: 5'-GACCTACGAGTCACCAAACAA-3' } \\
\text { R: } 5^{\prime} \text {-CACCTTCCGGGTCATAGAAAG-3' }\end{array}$ \\
\hline$\beta$-Actin & NM_031144 & $\begin{array}{l}\text { F: 5'-CTAAGGCCAACCGTGAAAAGAT-3' } \\
\text { R: } 5^{\prime} \text {-AGAGGCATACAGGGACAACACA-3' }\end{array}$ \\
\hline
\end{tabular}

$\mathrm{F}=$ Forward; $\mathrm{R}$ = reverse.

Image-Pro Analyzer 7.0 (Media Cybernetics Inc., Rockville, Md., USA) to quantitatively assess the degree of osteogenesis. Cells were also collected at 1 and 2 weeks, and total RNA was extracted for subsequent RT-PCR analysis to determine if BMP6 affects the expression of the osteogenic genes BSP and runt-related transcription factor 2 (Runx2).

The second experiment was conducted to determine the effect of endogenous BMP6 on the osteogenesis of DFSCs. For this experiment, a dicer substrate BMP6-siRNA (guide strand: $5^{\prime}$-rGrArArGrArArGrGrCrUrGrGrCrUrGrGrArArUrUrCrGrArCrA-3'; passenger strand: $5^{\prime}$-rUrGrUrCrGrArArUrUrCrCrArGrCrCrArGrCrCrUrUrCrUrUrCrGrG-3') was designed based on the mRNA sequence of rat BMP6 and synthesized by Integrated DNA Technologies Inc. (Coralville, Iowa, USA). DFSCs of passage 3 were transfected with the BMP6-siRNA using RNAiMAX ${ }^{\text {TM }}$ (Life Technologies, Carlsbad, Calif., USA). The control DFSCs were transfected with a scrambled siRNA or underwent mock transfection. Cells were collected at days 1,2, 3, 4 and 5 following transfection to determine the BMP6 knockdown efficiency by Western blotting. Transfected cells in a separate plate were subjected to osteogenic induction for 2 weeks with a repeated BMP6-siRNA transfection at day 7. After 2 weeks of osteogenic induction, the cells were fixed and stained with alizarin red to detect osteogenesis. The staining was quantified by Image-Pro Analyzer 7.0.

RT-PCR and Western Blot to Determine Gene Expression

For real-time RT-PCR, total RNA was isolated from the cells with an RNeasy mini kit (Qiagen, Valencia, Calif., USA). RNA concentration was measured with a Nanodrop spectrophotometer after DNase I treatment. Equal amounts of RNA were reversetranscribed into cDNA, and the cDNA was used for real-time PCR to determine the threshold cycle values with gene-specific primers. Relative gene expression (RGE) was calculated by the delta threshold cycle method using $\beta$-actin as the endogenous control. The primer pairs used for PCR analysis in this study are listed in table 1.

For Western blotting, cells were lysed with ice-cold lysis buffer containing $15 \mathrm{~mm}$ sodium chloride, $1 \%$ Triton X-100, 0.1\% SDS, $50 \mathrm{mM}$ Tris and protease inhibitors. The cell lysate was centrifuged at $16,000 \mathrm{~g}$ for $15 \mathrm{~min}$, and the supernatant containing proteins was transferred into a fresh tube. Total protein was measured using the bicinchoninic acid method (Pierce, Rockford, Ill., USA), and $10 \mu \mathrm{g}$ of the protein was loaded to SDS-PAGE gel for electrophoresis. The protein was then transferred onto a polyvinylidene difluoride membrane. Following incubation of the membrane in 5\% skim milk for 30 min to reduce nonspecific binding, the membrane was stained with mouse monoclonal primary antibodies reactive to rat BMP6 (ab15640, Abcam, Cambridge, Mass., USA) and rat $\beta$-actin (A5441, Sigma-Aldrich, St. Louis, Mo., USA) for 3 h at room temperature or at $4{ }^{\circ} \mathrm{C}$ overnight. The membrane was incubated with goat anti-rabbit IgG conjugated with horseradish peroxidase. Finally, protein was detected with an enhanced chemiluminescence detection method, and imaging was captured with ChemiDoc (Bio-Rad, Hercules, Calif., USA).

\section{Statistical Analysis}

Results were reported as means \pm standard error. A Student $t$ test was used for statistical analysis to obtain the $\mathrm{p}$ values between two means for determining the significant differences in RGE between control and treatment, as well as in osteogenic quantitation between BMP6-treated and nontreated DFSCs. For comparison of RGE of BMP6 in the different passages of DFSCs and the effect of BMP6 siRNA knockdown on osteogenesis, analysis of variance and least significant difference were conducted with SAS programs, and means were considered to be significantly different at $\mathrm{p} \leq 0.05$.

\section{Results}

\section{Culture and Osteogenic Differentiation of DFSCs}

When the established DFSCs of passage 3 were incubated in osteogenic differentiation medium (DMEM + $10 \%$ FBS + osteogenic induction reagents) for about 2 weeks, they were capable of forming nodular deposits that could be stained with alizarin red (fig. 1a). In contrast, when the cells were cultured in DMEM without osteogenic induction reagents, no deposits were observed and 
Table 2. RGE of osteoblast and cementoblast markers during differentiation induction of DFSCs

\begin{tabular}{|c|c|c|c|c|c|c|}
\hline Culture medium & \multicolumn{2}{|l|}{$\mathrm{OCN}$} & \multicolumn{2}{|l|}{ BSP } & \multicolumn{2}{|l|}{ F-Spondin } \\
\hline Basal medium + induction reagents & $3.8 \pm 1.2$ & $278.7 \pm 41.2^{* *}$ & $174.8 \pm 55.8^{*}$ & $403.4 \pm 84.8^{* *}$ & $0.94 \pm 0.28$ & $0.1 \pm 0.01^{* *}$ \\
\hline
\end{tabular}

RGE values are reported as means \pm SE $(n=4) .{ }^{*} \mathrm{p} \leq 0.05,{ }^{* *} \mathrm{p} \leq 0.01$ : significantly different from control.
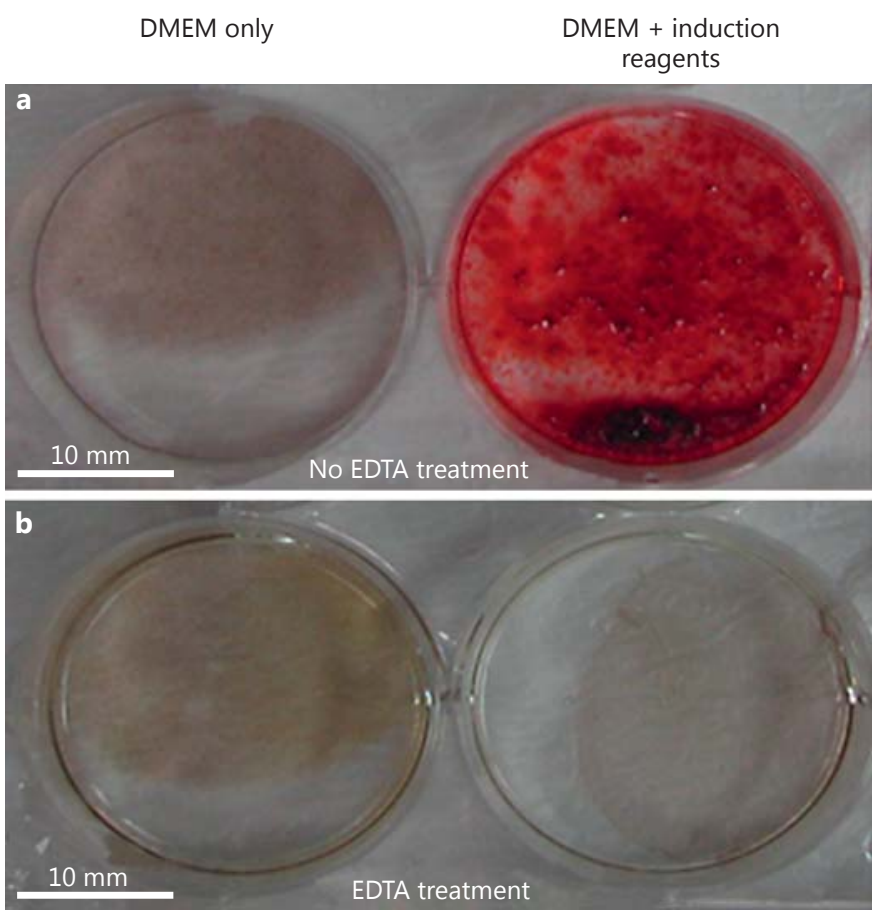

Fig. 1. Osteogenic induction of DFSCs resulted in formation of calcium deposits as determined by alizarin red staining. a Alizarin red-positive staining was seen only when cells were cultured in DMEM supplemented with induction reagents, and no alizarin red staining was seen in the DFSCs cultured in DMEM without induction reagents. b Treatment of the cultures with 10\% EDTA prior to alizarin red staining completely removed the deposits and resulted in no staining in the induced cells, similar to the noninduced cells.

alizarin red staining was negative (fig. 1a). To determine if the deposits were calcium, some wells were treated with $10 \%$ EDTA prior to alizarin red staining, and we found that the deposits could be completely removed by EDTA treatment such that no alizarin red staining could be seen in EDTA-treated wells (fig. 1b), indicating that these deposits were calcium.

Expression and Role of BMP6 in Dental Follicle Stem Cells
Because the DF is believed to harbor cells for development of the cementum and alveolar bone, and alizarin red staining cannot distinguish if the calcium depositions are from osteoblasts or cementoblasts, we examined the expression of osteoblast and cementoblast markers in the DFSCs subjected to differentiation induction. Expression of osteoblast markers (BSP and OCN) and a cementoblast marker (F-spondin) was determined with real-time RTPCR. The DFSCs cultured in DMEM (basal medium) without osteogenic induction reagents expressed minimal OCN and BSP, whereas an average 3.8-fold increase in OCN expression and 174.8-fold increase in BSP were seen after 1 week of osteogenic induction. Expression of the markers continued to increase to an average of 278.7fold for OCN and 403.8-fold for BSP after 2 weeks of induction (table 2). In contrast, expression of F-spondin was decreased by about $90 \%$ (i.e. RGE of 0.1 ) after 2 weeks of induction (table 2). The changes in the expression of all these marker genes were statistically significant after 2 weeks of induction at $\mathrm{p} \leq 0.05$. F-Spondin has been reported to increase expression in human cementoblastlike cells and was identified as a promoting factor for cementoblastic differentiation [Kitagawa et al., 2006]. Increased expression of the osteoblast markers and decreased expression of the cementoblast marker, together with calcium depositions seen with alizarin red staining, suggested that the DFSCs differentiated toward osteoblasts but not cementoblasts under our osteogenic induction conditions.

Comparison of BMP6 Expression in DFCs and DFSCs

The expression of BMP6 in DFCs and DFSCs was compared when both cell types were grown in a-MEM + $20 \%$ FBS. Real-time RT-PCR showed that BMP6 was highly expressed in the DFSCs. On average, BMP6 expression in the DFSCs was about 17-fold higher than in the DFCs (fig. 2a). Statistical analysis indicated that this difference was highly significant $(\mathrm{p}=0.001)$. Western blotting showed that both cell types produced BMP6 pro- 
Fig. 2. Comparison of BMP6 expression in DFCs and DFSCs as determined by realtime RT-PCR (a) and Western blotting (b). Both analyses indicated that DFSCs expressed a higher level of BMP6 than the DFCs did. BMP6 expression in the DFSCs was 17 times greater than that in the DFCs as determined by the RGE in real-time RTPCR analysis (** $\mathrm{p} \leq 0.01 ; \mathrm{n}=4)$.

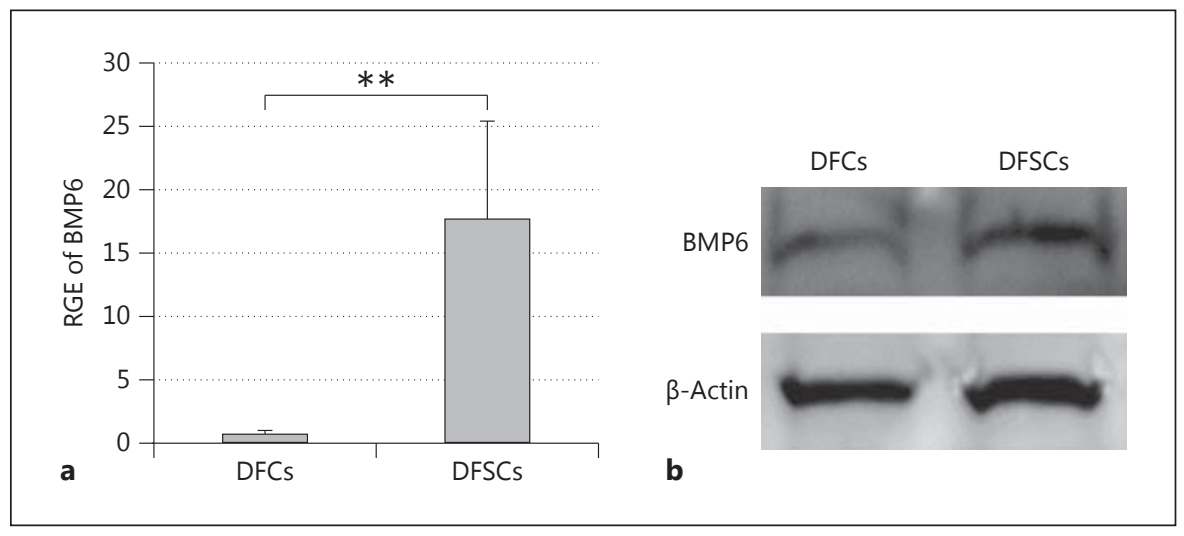

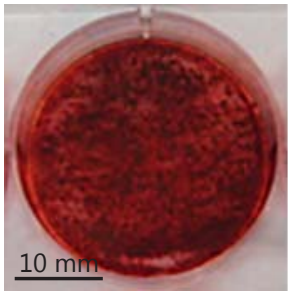

Passage 3

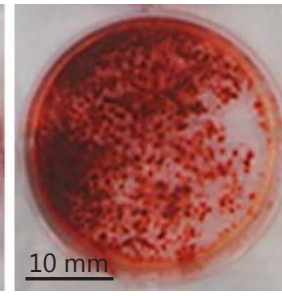

Passage 5

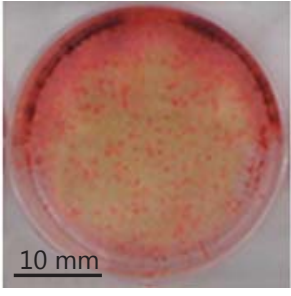

Passage 7

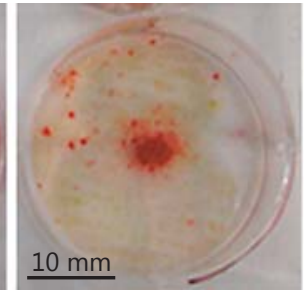

Passage 9

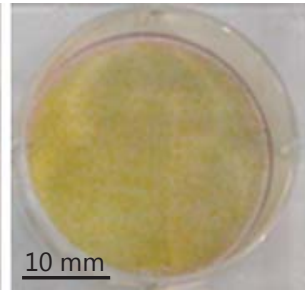

Passage 11

a
Fig. 3. Evaluation of differentiation potential and BMP6 expression in different passages of DFSCs. a Osteogenic differentiation of different passages of DFSCs revealed reduction of the osteogenic capability in later passages as shown by alizarin red staining of calcium deposits. b Expression of BMP6 in different passages of DFSCs during osteogenic differentiation indicated that DFSCs expressed significantly higher levels of BMP6 in the early passages than in late passages as determined by real-time RT-PCR. Bars labeled with the same letter indicate no statistical difference in RGE at $\mathrm{p} \leq 0.05(\mathrm{n}=4) . \mathrm{P}=$ Passage.

tein, but DFSCs produced a higher amount of BMP6 protein than did the DFCs (fig. 2b) under the same culture conditions, which was consistent with the real-time RTPCR results.

\section{Osteogenic Capability of DFSCs during in vitro \\ Proliferation}

When different passages of DFSCs were subjected to osteogenic induction for 2 weeks, maximum calcium de-

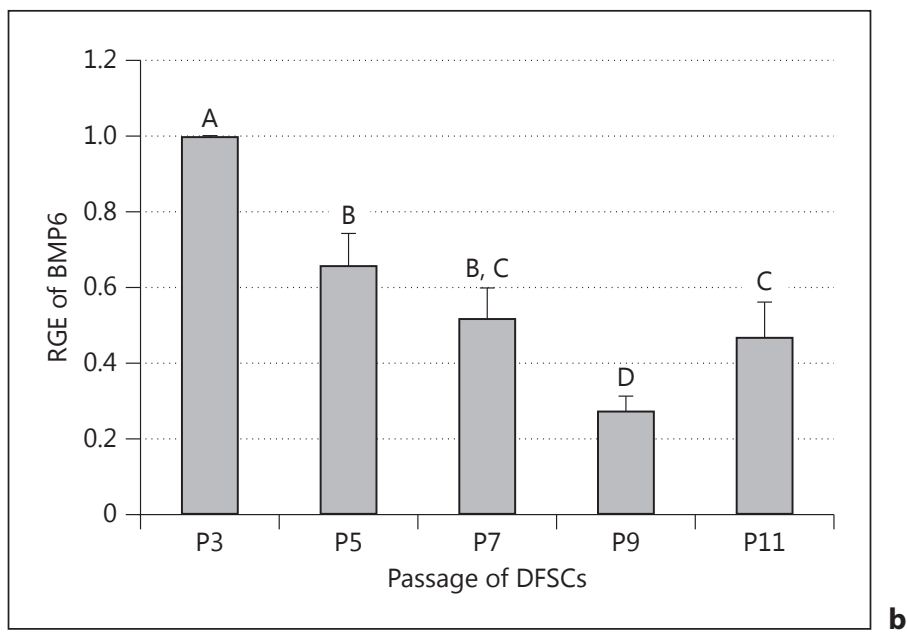

position occurred in the DFSCs at passages 3 and 5, as revealed by alizarin red staining. A dramatic reduction in alizarin red staining was seen at passage 7 . The staining was further reduced in passage 9 cells. In passage 11 cells, alizarin red staining could only be seen occasionally (fig. 3a). The results indicated that the DFSCs reduced their osteogenic capability during in vitro culture, and complete loss of this ability occurred around passage 11 . 


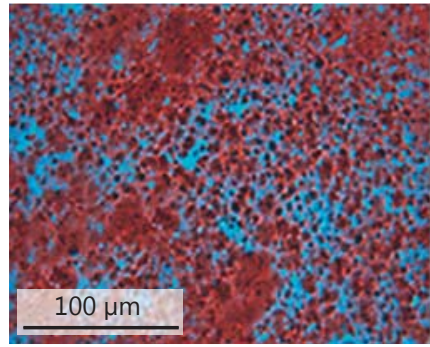

P3 DFSCs

(Control)

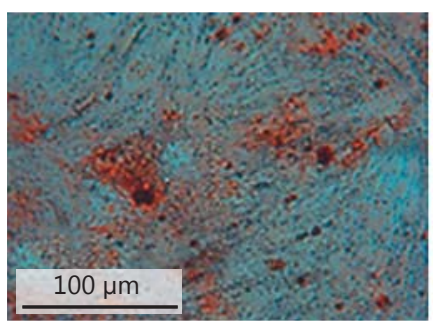

P7 DFSCs

(Control)

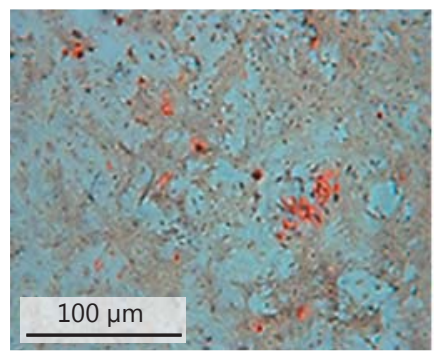

P11 DFSCs

a

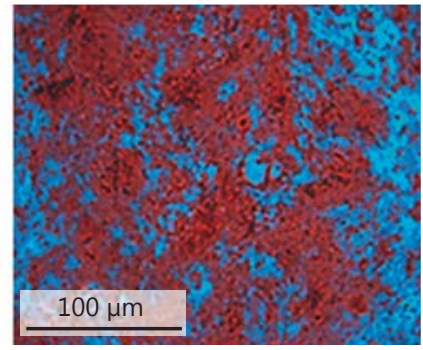

P3 DFSCs +

$80 \mathrm{ng} / \mathrm{ml} \mathrm{hrBMP6}$

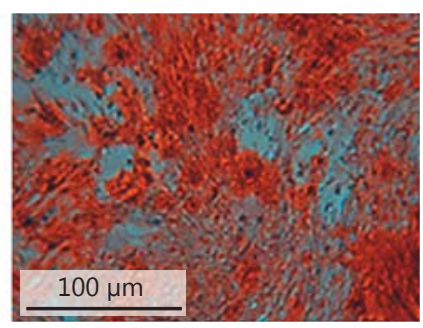

P7 DFSCs +

$80 \mathrm{ng} / \mathrm{ml}$ hrBMP6

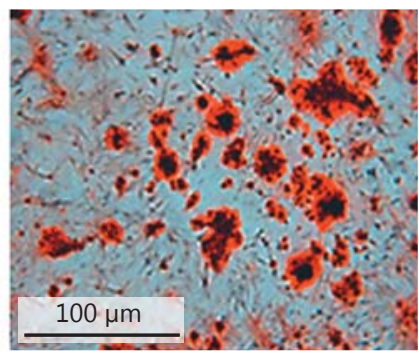

P11 DFSCs +

$80 \mathrm{ng} / \mathrm{ml} \mathrm{hrBMP6}$

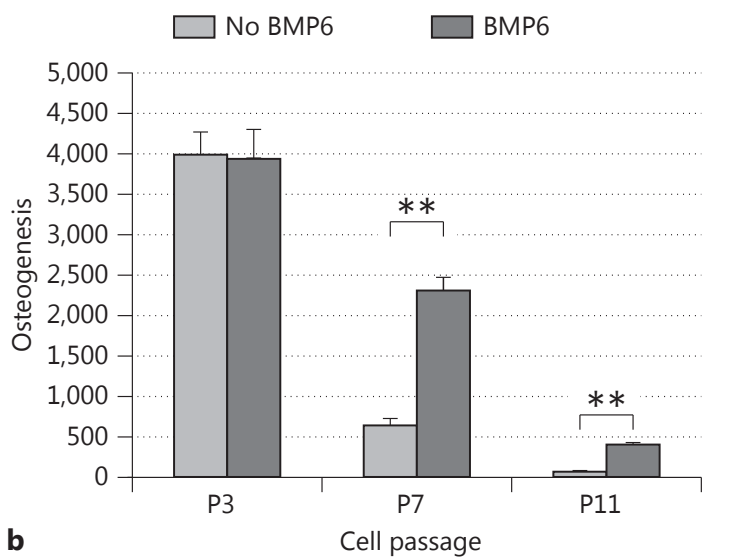

b

Cell passage

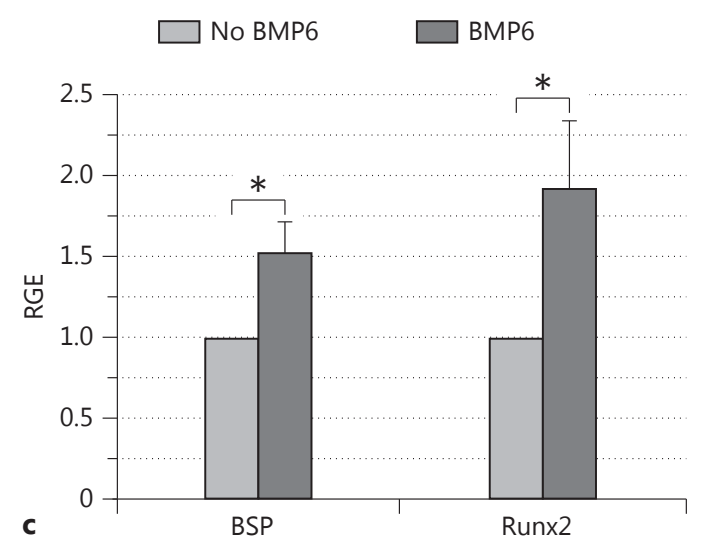

Fig. 4. Effect of exogenous BMP6 on osteogenesis of DFSCs. a Addition of hrBMP6 to the osteogenic induction medium resulted in no obvious effect on osteogenesis in passage 3 (P3) DFSCs but dramatically increased osteogenesis in passage 7 (P7) and 11 (P11) DFSCs, as shown by alizarin red staining of calcium deposits. b Quantitation of osteogenesis by analyzing alizarin red staining using Image-Pro Analyzer. Statistical analysis of the results showed

Expression of BMP6 in Different Passages of DFSCs

The above experiment showed that the cultured DFSCs had reduced osteogenic capability with advancement of cell passaging. To determine if any changes in BMP6 expression occurred in later passages of DFSCs during osteogenic induction, different passages of DFSCs were placed in osteogenic induction medium for 1 week and collected for real-time RT-PCR analysis. Maximal BMP6 expression was seen in the DFSCs of passage 3. BMP6 expression was a significant increase in osteogenesis by adding hrBMP6 to the medium for osteogenic induction of passage 7 and 11 DFSCs. ** $\mathrm{p} \leq 0.01(\mathrm{n}=3)$. c Expression of BSP and Runx2 in passage 7 DFSCs after 2 weeks of osteogenic induction as determined by real-time RT-PCR. Increased expression of BSP and Runx2 was detected in the DFSCs when hrBMP6 was added to the induction medium. ${ }^{*} \mathrm{p} \leq 0.05(\mathrm{n}=3)$. decreased in other passages of DFSCs. Generally, the higher the cell passage, the lower the BMP6 expression observed. On average, BMP6 expression at passage 7 was decreased by $50 \%$ compared to passage 3 . The expression further reduced to $25 \%$ of that of passage 3 at passage 9 (fig. 3b). This reduction in BMP6 expression seen in the late passages was statistically significant at $\mathrm{p} \leq 0.05$. 

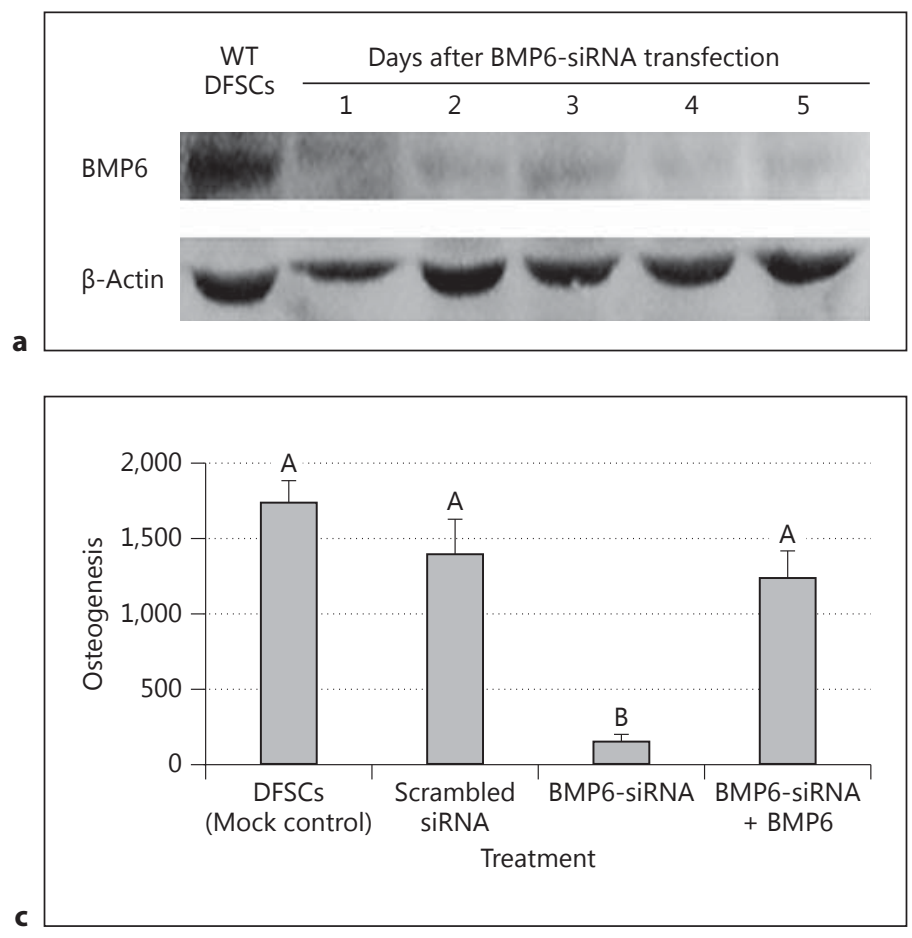

Fig. 5. Effect of BMP6 knockdown on osteogenesis of DFSCs. a BMP6 expression was greatly knocked down by BMP6-siRNA as determined by Western blotting. WT = Wild-type. $\mathbf{b}$ Knockdown of BMP6 reduced the osteogenic differentiation capability of DFSCs as seen by comparing the BMP6-siRNA-transfected DFSCs with the controls (untransfected and scrambled siRNA-transfected DFSCs). Incubation of BMP6-siRNA-transfected cells in osteo-

\section{Effect of BMP6 on Osteogenesis of DFSCs}

To further study the role of BMP6 on osteogenesis of DFSCs, hrBMP6 was added to the medium for induction of osteogenesis. The results showed that DFSCs at passage 3 possess strong osteogenesis regardless of the presence of hrBMP6 in the osteogenic induction medium; i.e., no obvious effect of hrBMP6 was observed for osteogenic induction of the DFSCs at passage 3 (fig. $4 a, b$ ). In contrast, when hrBMP6 was added to the osteogenic medium for osteogenesis of DFSCs of passages 7 and 11, the passages in which the osteogenic capability and BMP6 expression were greatly reduced as compared to the passage 3 DFSCs, a significant increase in osteogenesis was observed with hrBMP6 treatment as compared to the control without hrBMP6 after induction (fig. 4a, b). We noticed that such an effect of BMP6 on osteogenic differentiation of DFSCs was clearly shown after 2 weeks of induction for passage 3 and passage 7 DFSCs. However, for passage 11 DFSCs, 3 weeks of induction was needed

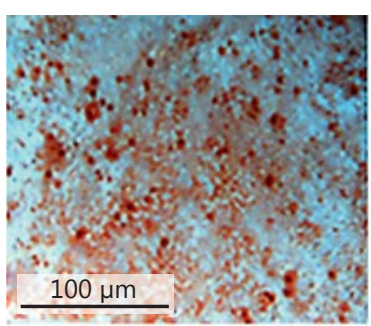

DFSCs only

(Mock control)

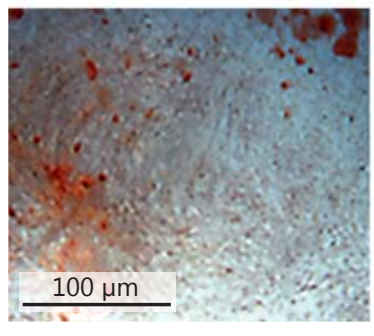

BMP6-siRNA

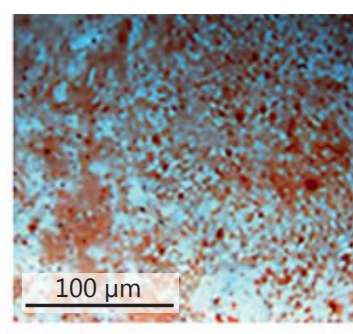

Scrambled siRNA (Control)

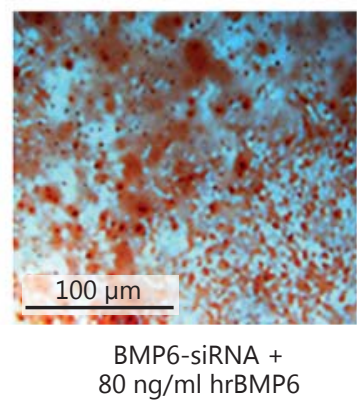

genic medium containing hrBMP6 protein restored the osteogenesis (lower right panel).c Osteogenesis was quantified by analyzing alizarin red staining using Image-Pro Analyzer. Statistical analysis indicated a significant reduction in osteogenesis after BMP6 knockdown, and addition of hrBMP6 could significantly restore osteogenesis $(n=4)$. Bars labeled with the same letter indicate no significant difference at $\mathrm{p} \leq 0.05$.

to show osteogenesis and an obvious BMP6 effect as seen in figure 4. Furthermore, real-time RT-PCR analysis showed that BMP6 treatment significantly increased the expression of the osteogenic genes BSP and Runx 2 in passage 7 DFSCs (fig. 4c).

To determine if a reduction in BMP6 expression affects osteogenesis, expression of BMP6 in the early-passage DFSCs was knocked down by BMP6-siRNA transfection. The knockdown effect appeared to last for at least 5 days, as determined by Western blotting analysis (fig. 5a). When the DFSCs with BMP6 knockdown were subjected to osteogenic induction, a dramatic reduction in osteogenesis was observed as compared to the mock transfection control or scrambled siRNA transfection control using alizarin red staining (fig. 5b). The osteogenesis was quantified by analyzing the staining with an Image-Pro Analyzer. Knockdown of BMP6 expression resulted in about $90 \%$ reduction of osteogenesis as compared to the controls. Statistical analysis indicated that 
the reduction was statistically significant (fig. 5c). Moreover, this reduction of osteogenesis by BMP6 knockdown could be restored by addition of exogenous hrBMP6 protein to the osteogenic induction medium (fig. 5b, c).

\section{Discussion}

DFSCs have been shown to possess multilineage differentiation capability [Yao et al., 2008]. When DFSCs were cultured in medium without induction reagents (i.e. without ascorbate- 2 phosphate, dexamethasone and $\beta$ glycerophosphate), they did not form calcium deposits (fig. 1a), indicating that the cell population contained no calcium-depositing cells such as osteoblasts and cementoblasts. When the induction reagents were included in the medium, calcium deposits were observed and expression of BSP and OCN (osteoblast markers) was dramatically increased, but no significantly increased expression of F-spondin (cementoblast marker) was observed (table 2). In fact, decreased expression of F-spondin was seen after 2 weeks of induction. These results suggested that the DFSCs were induced to differentiate into osteoblasts but not cementoblasts.

The DFSCs used in this study were maintained in medium containing $20 \%$ FBS. FBS at a concentration of 10 $20 \%$ remains a common standard medium supplement in culturing mesenchymal stem cells for basic research, as well as for clinical studies [see review by Jung et al., 2012], although there are some concerns with using FBS in cell culture, including the concern that FBS can spontaneously induce osteogenic differentiation of stem cells. The results of this study revealed that the osteogenic induction reagents had to be included in the medium for induction of differentiation as shown by calcium deposits and increased expression of BSP and OCN (fig. 1; table 2), suggesting that FBS alone cannot spontaneously induce osteogenic differentiation of DFSCs. Our observation was in agreement with other published results. A study by Shahdadfar et al. [2005] also showed that human mesenchymal stem cells maintained in FBS did not form calcium deposits without proper induction with the above-mentioned induction reagents. Moreover, another study demonstrated that mesenchymal stem cells grown in FBS-containing medium remained undifferentiated and that the FBS concentration did not affect differentiation [Gong et al., 2009].

Although all experiments were conducted with FBS purchased from Atlanta Biologicals, different lots of FBS were used. In that vein, we observed variations in osteo-

Expression and Role of BMP6 in Dental Follicle Stem Cells genesis in given passages of DFSCs from experiment to experiment. It is possible that the variations were caused by different lots of FBS. Variation of differentiation in given passages was also seen when using different batches of DFSCs with the same lot of FBS, suggesting that DFSCs isolated from different litters may vary in their differentiation capability. However, the trend of loss of differentiation with advancement of passages was consistent regardless of whether different lots of FBS or DFSCs derived from different litters were used.

BMP6 has been shown to stimulate new bone formation in vivo [Simic et al., 2006]. Recently, we have reported that BMP6 is expressed in the DF of postnatal rat pups and its expression displays a continuous increase in the DF through day 11 postnatally [Wise et al., 2011]. Knockdown of BMP6 by siRNA did result in reduction of bone formation in the base of the tooth crypt and tooth impaction, suggesting the vital role of BMP6 in tooth eruption [Wise et al., 2011]. We believe this is due to the effect of BMP6 in regulating osteogenic differentiation. In the current study, we compared the BMP6 expression in DF-derived cells, namely DFCs and DFSCs, and found that both types of cells produce BMP6, but DFSCs expressed a significantly higher amount of BMP6 than did the DFCs under the same culture conditions (fig. 2). However, this does not imply that DFSCs are the major BMP6 producer in the DF in vivo, as the majority of cells in the DF are fibroblast-like cells (i.e. DFCs), and stem cells only comprise a small portion. BMP6 is a secreted molecule [Rickard et al., 1998], and BMPs exert their biological effect via binding to their receptors on cell membranes, including activin receptor-like kinase-2 and BMP type II receptor [Ebisawa et al., 1999]. In a study to determine the role of BMPs in the synthesis of follicle-stimulating hormone, BMP6 has been demonstrated to exert autocrine and paracrine regulation [Huang et al., 2001]. High-level expression of BMP6 in the DFSCs may favor them to undergo osteogenic differentiation by the autocrine pathway. This is supported by the in vitro osteogenic experiments showing that the osteogenic potential of DFSCs was correlated to BMP6 expression (fig. 3), and knockdown of endogenous BMP6 reduced the osteogenesis of DFSCs (fig. 5). In addition, DFCs also produced BMP6, although in lesser amounts (fig. 2). Thus, BMP6 secreted by DFCs may serve as a supplemental source of BMP6 to upregulate osteogenesis of DFSCs via the paracrine pathway. Such osteogenesis of DFSCs might contribute to the overall bone growth seen in vivo in the tooth crypt.

Furthermore, DFSCs may also contribute to bone formation by secreting and transporting BMP6 to the adja- 
cent osteoblasts in alveolar bone, which could accelerate bone growth by stimulating the osteoblasts for mineralization. This is supported by the observation that BMP6 treatment promoted the formation of mineralized nodules in human primary osteoblasts [Grasser et al., 2007]. In addition, BMP6 produced by both DFSCs and normal DFCs might stimulate osteogenesis of stem cells in alveolar bone. Other studies have revealed that when bone marrow-derived mesenchymal stem cells were induced for osteogenic differentiation in a medium containing exogenous BMP6, increased osteogenesis was observed [Lavery et al., 2008]. This study showed that incubation of DFSCs in medium containing BMP6 could increase expression of BSP and Runx2 genes (fig. 4c), which may promote osteogenic differentiation.

The in vitro osteogenic induction experiments demonstrated that addition of exogenous BMP6 could upregulate osteogenesis of DFSCs only when BMP6 expression was reduced in the late-passage cells (fig. $4 a, b)$ or when BMP6 expression was knocked down by siRNA (fig. 5b, c). However, when BMP6 protein was added into the medium for osteogenic induction of the early-passage DFSCs, which express a high level of BMP6, no further increase in osteogenesis was observed (fig. 4a, b). We reasoned that this is because the early-passage DFSCs produced a maximal amount of endogenous BMP6 (fig. 3), which was sufficient to mask the effect of exogenous BMP6. Therefore, addition of exogenous BMP6 resulted in no effect for induction of osteogenesis of DFSCs. Given that BMP6 is the most potent regulator of osteoblast differentiation of mesenchymal stem cells [Friedman et al., 2006], there is likely a threshold beyond which additional BMP6 is not effective.

Loss of differentiation capability is a common phenomenon during in vitro expansion of adult stem cells, as seen in dental pulp stem cells [Takeda et al., 2008]. This study clearly showed that DFSCs gradually lose their differentiation capability during in vitro culture (fig. 3a). This loss of differentiation capability during in vitro expansion hampers utilization of the stem cells for regenerative medicine. However, the reasons or mechanisms causing the stem cells to lose their differentiation capability have not been elucidated. Here, we found that when different passages of DFSCs were subjected to osteogenic induction, the early-passage DFSCs (e.g. passage 3) expressed maximal levels of BMP6. In contrast, when the late passages of DFSCs were induced for osteogenesis, they expressed significantly lower BMP6 than did the early-passage DFSCs (fig. 3b), suggesting that high-level expression of BMP6 is likely important for DFSCs to un- dergo osteogenesis. The failure to express sufficient BMP6 during osteogenic differentiation in the late passages of DFSCs may be responsible, at least partially, for their reduction or loss of osteogenic capability.

With this in mind, we conducted a BMP6 knockdown study. Knockdown of BMP6 expression by BMP6-siRNA in the early-passage DFSCs greatly reduced their osteogenic capability. Importantly, the reduction of osteogenic capability by siRNA could be restored by addition of exogenous hrBMP6 (fig. 5). Together with the observation that the loss of osteogenic capability in late-passage DFSCs could be restored by exogenous BMP6 (fig. 4a), we conclude that low-level BMP6 expression in late passages of DFSCs during osteogenic induction might contribute to the loss or reduction of osteogenesis.

BMP6 knockdown dramatically reduced osteogenic differentiation of DFSCs but did not completely inhibit osteogenesis. We speculate that this is because (1) expression of BMP6 is greatly reduced, but not completely eliminated by siRNA transfection, and (2), more likely, BMP6 is not the only factor regulating osteogenesis. Other osteogenic genes such as BMP2, BMP3 and BSP are also expressed in DF and DFSCs (data not shown). These factors may contribute to the osteogenic differentiation of DFSCs. Future studies may determine the role of these factors/genes in regulating DFSC differentiation and elucidate if BMP6 and those factors have an additive or synergistic effect. We showed that addition of hrBMP6 protein to the culture of passage 11 DFSCs, whose endogenous BMP6 expression was greatly reduced, partially recovered osteogenesis (fig. 4a, b), supporting the idea that BMP6 may not be the sole requirement for osteogenesis of DFSCs.

In conclusion, both DFCs and DFSCs express BMP6, which would contribute to the overall BMP6 production in vivo in the DF. DFSCs produce a higher amount of BMP6 than do DFCs. High-level expression of BMP6 appeared to be important for osteogenic differentiation of DFSCs, and the low level of BMP6 expression in late passages may explain the reduction of osteogenesis in these passages. Increased expression of BMP6 seen in the DF would likely favor the osteogenesis of DFSCs as well as the osteogenesis of cells in the alveolar bone adjacent to the DF.

\section{Acknowledgements}

This research was supported by National Institute of Dental and Craniofacial Research grant 5R01DE008911-21 to G.E.W. and S.Y. The authors would like to thank Dr. Xiaochu Wu and $\mathrm{Mr}$. Gregory McCormick for their assistance in using Image-Pro Analyzer for quantification of osteogenesis. 


\section{References}

Ebisawa. T., K. Tada, I. Kitajima, K. Tojo, T.K. Kemmis, C.M., A. Vahdati, H.E. Weiss, D.R. Sampath, M. Kawabata, K. Miyazono, T. Imamura (1999) Characterization of bone morphogenetic protein-6 signaling pathways in osteoblast differentiation. J Cell Sci 112: 3519-3527.

Friedman, M.S., M.W. Long, K.D. Hankenson (2006) Osteogenic differentiation of human mesenchymal stem cells is regulated by bone morphogenetic protein-6. J Cell Biochem 98: 538-554.

Gong, Z., G. Calkins, E.C. Cheng, D. Krause, L.E. Niklason (2009) Influence of culture medium on smooth muscle cell differentiation from human bone marrow-derived mesenchymal stem cells. Tissue Eng Part A 15: 319-330.

Grasser, W.A., I. Orlic, F. Borovecki, K.A. Riccardi, P. Simic, S. Vukicevic, V.M. Paralkar (2007) BMP-6 exerts its osteoinductive effect through activation of IGF-I and EGF pathways. Int Orthop 31: 759-765.

-Gruber, R., W. Graninger, K. Bobacz, G. Watzek, L. Erlacher (2003) BMP-6-induced osteogenic differentiation of mesenchymal cell lines is not modulated by sex steroids and resveratrol. Cytokine 23: 133-137.

Honda, M.J., M. Imaizumi, S. Tsuchiya, C. Morsczeck (2010) Dental follicle stem cells and tissue engineering. J Oral Sci 52: 541-552.

Huang, H.J., J.C. Wu, P. Su, O. Zhirnov, W.L. Miller (2001) A novel role for bone morphogenetic proteins in the synthesis of folliclestimulating hormone. Endocrinology 142: 2275-2283.

-Jung, S., K.M. Panchalingam, L. Rosenberg, L.A. Behie (2012) Ex vivo expansion of human mesenchymal stem cells in defined serumfree media. Stem Cells Int 2012: 123030. Wagner (2010) Bone morphogenetic protein 6 drives both osteogenesis and chondrogenesis in murine adipose-derived mesenchymal cells depending on culture conditions. Biochem Biophys Res Commun 401: 20-25.

Kitagawa, M., Y. Kudo, S. Iizuka, I. Ogawa, Y. Abiko, M. Miyauchi, T. Takata (2006) Effect of F-spondin on cementoblastic differentiation of human periodontal ligament cells. Biochem Biophys Res Commun 349: 10501056.

Lavery, K., P. Swain, D. Falb, M.H. Alaoui-Ismaili (2008) BMP-2/4 and BMP-6/7 differentially utilize cell surface receptors to induce osteoblastic differentiation of human bone marrow-derived mesenchymal stem cells. J Biol Chem 283: 20948-20958.

Marks, S.C., Jr., D.R. Cahill (1987) Regional control by the dental follicle of alterations in alveolar bone metabolism during tooth eruption. J Oral Pathol 16: 164-169.

Rickard, D.J., L.C. Hofbauer, S.K. Bonde, F. Gori, T.C. Spelsberg, B.L. Riggs (1998) Bone morphogenetic protein- 6 production in human osteoblastic cell lines. Selective regulation by estrogen. J Clin Invest 101: 413-422.

Sappino, A.P., W. Schürch, G. Gabbiani (1990) Differentiation repertoire of fibroblastic cells: expression of cytoskeletal proteins as marker of phenotypic modulations. Lab Invest 63 : 144-161.

Shahdadfar, A., K. Frønsdal, T. Haug, F.P. Reinholt, J.E. Brinchmann (2005) In vitro expansion of human mesenchymal stem cells: choice of serum is a determinant of cell proliferation, differentiation, gene expression, and transcriptome stability. Stem Cells 23: 1357-1366.
Simic, P., J.B. Culej, I. Orlic, L. Grgurevic, N. Draca, R. Spaventi, S. Vukicevic (2006) Systemically administered bone morphogenetic protein- 6 restores bone in aged ovariectomized rats by increasing bone formation and suppressing bone resorption. J Biol Chem 281: 25509-25521.

Takeda, T., Y. Tezuka, M. Horiuchi, K. Hosono, K. Lida, D. Hatakeyama, S. Miyaki, T. Kunisada, T. Shibata, K. Tezuka (2008) Characterization of dental pulp stem cells of human tooth germs. J Dent Res 87: 676-681.

Wise, G.E., H. He, D.L. Gutierrez, S. Ring, S. Yao (2011) Requirement of alveolar bone formation for eruption of rat molars. Eur J Oral Sci 119: 333-338.

Wise, G.E., G.J. King (2008) Mechanisms of tooth eruption and orthodontic tooth movement. J Dent Res 87: 414-434.

Wise, G.E., F. Lin, W. Fan (1992) Culture and characterization of dental follicle cells from rat molars. Cell Tissue Res 267: 483-492.

Wise, G.E., S. Yao (2006) Regional differences of expression of bone morphogenetic protein-2 and RANKL in the rat dental follicle. Eur J Oral Sci 114: 512-516.

Wise, G.E., S. Yao, W.G. Henk (2007) Bone formation as a potential motive force of tooth eruption in the rat molar. Clin Anat 20: 632639

Yao, S., F. Pan, V. Prpic, G.E. Wise (2008) Differentiation of stem cells in the dental follicle. J Dent Res 87: 767-771.

Yao, S., V. Prpic, F. Pan, G.E. Wise (2010) TNFalpha upregulates expression of BMP-2 and BMP-3 genes in the rat dental follicle - implications for tooth eruption. Connect Tissue Res 51: 59-66. 